\title{
The interaction of nitric oxide, bradykinin, and the angiotensin II type 2 receptor: lessons learned from transgenic mice
}

Commentary

See related article in vol. 104 no. 7 , pages 925-935.

\author{
Charles D. Searles and David G. Harrison \\ Division of Cardiology, Department of Medicine, Emory University School of Medicine, Atlanta, Georgia 30322, USA
}

Address correspondence to: Charles D. Searles, Department of Medicine, Emory University, 1639 Pierce Drive, Division of Cardiology, Atlanta, Georgia 30322, USA. Phone: (404) 727-3138; Fax: (404) 727-3330; E-mail: csearle@emory.edu.

\begin{abstract}
Despite intensive study over the past few years, several aspects of the reninangiotensin system remain poorly understood. Such is the case for the angiotensin II type 2 receptor (AT2), one of the two receptor subtypes that mediate the actions of angiotensin II. In fetal tissues, this receptor is expressed at high levels and appears to have a role in growth, differentiation, and maturation of cells in various organs, including the vasculature (1-4). As development progresses, the level of expression of the AT2 is downregulated. In adult animals, in contrast to the wide distribution of angiotensin II type 1 receptor (AT1), AT2 is expressed at low levels and is restricted to the adrenal gland, brain, ovary, uterus, kidney, and heart. Importantly, there appears to be upregulation of AT2 in pathological states such as salt depletion, heart failure, experimental cardiac hypertrophy, myocardial infarction, and vascular injury (3-8).

Because of the low level of AT2
\end{abstract} expression in normal tissues, there is substantial debate as to its role under normal circumstances. A growing body of research suggests that there is crosstalk between AT1 and AT2 in mediating the physiologic effects of angiotensin II. Based largely on pharmacological studies, stimulation of the AT2 seems to antagonize several of the effects caused by AT1 stimulation. Thus, AT1 stimulation activates the mitogen-activated protein kinases ERK1 and ERK2, whereas AT2 stimulation suppresses this pathway, perhaps by activating ERK phosphatase 1 (MKP-1) (4, 9, 10). AT1 stimulation promotes cellular growth and hypertrophy, while the AT2 antagonizes them (3-6). AT1 stimulation facilitates angiogenesis, while the AT2 inhibits this process (11). AT1 activation induces vasoconstriction, while AT2 activation causes vasodilation (12). These cellular and organ-level effects appear to act in intact animal models as well. In cardiomyopathic hamsters, AT2 expression is upregulated in cardiac fibroblasts of the failing heart and appears to antagonize AT1-mediated progression of interstitial fibrosis and cardiac remodeling (6). Overexpression of AT2 in balloon-injured vascular smooth muscle cells attenuates neointimal formation (6). In a rat model of ischemic cardiomyopathy, the beneficial effects of AT1 blockade on cardiac remodeling and hemodynamics are inhibited by AT2 blockade (5).

Recent work has suggested that some of the beneficial effects of AT2 stimulation may be meditated through the bradykinin/nitric oxide (NO) cascade (5, 13-15). Endothelial cells contain bradykinin type 2 receptors $\left(B_{2}\right)$, which, when activated, potently stimulate production of NO. Although the effects of angiotensin II and NO seem to vary with the concentration of $\mathrm{NO}$ and the cell type involved, these two factors appear to play opposing roles in the cardiovascular system: angiotensin II is a potent stimulus for vasoconstriction and vascular smooth muscle hypertrophy, whereas NO has a vasodepressor effect and has been shown to be an antiproliferative agent. Thus, in spontaneously hypertensive rats, AT2 activation has been shown to increase vascular cyclic guanosine 3',5'-monophosphate (cGMP) levels, an effect that could be inhibited by $\mathrm{B}_{2}$ blockade or by inhibition of NO synthase (15). Likewise in conscious rats, salt depletion, which activates the renin-angiotensin system, increases cGMP levels in the renal interstitial fluid (14), an effect that can be prevented by blockade of either NO synthase or the AT2. Following myocardial infarction in rats, either angiotensin I-converting enzyme inhibitors or AT1 antagonists can prevent remodeling of the left ventricle, as assessed by collagen deposition, myocyte size, and left ventricular diameter (5), and in either case the effect could be blocked by $\mathrm{B}_{2}$ inhibition. Importantly, these studies not only underscore the antagonistic interactions between AT1 and AT2 but also introduce the notion that the bradykinin/NO system mediates this interaction.

In the previous issue of the JCI, Tsutsumi et al. (16) provided additional compelling evidence linking AT2 to the bradykinin/NO cascade. These authors targeted overexpression of AT2 to the vascular smooth muscle in transgenic mice, achieving a 5-fold increase in expression of this receptor. These animals exhibited an attenuated pressor response to angiotensin II infusion. Pretreatment of these transgenic mice with an AT2 antagonist, a $B_{2}$-receptor antagonist, or an NO synthase inhibitor restored the pressor response to angiotensin II. Angiotensin II produced a paradoxical decrease in blood pressure after AT1 blockade in these animals, suggesting that selective AT2 stimulation had a vasodepressor effect. Furthermore, the authors showed that the AT2-mediated vasodepressor effect was associated with an endotheliumdependent increase in aortic production of cGMP and activation of the kininkallikrein system. In the authors' interpretation of the results, angiotensin II stimulates AT2 in vascular smooth muscle, which leads to activation of the kinin-kallikrein system and bradykinin release. Bradykinin then binds to its receptor on adjacent endothelial cells, causing the release of $\mathrm{NO}$ and stimulation of cGMP.

As with other work investigating the physiologic role of AT2, this study 
relied heavily on pharmacologic manipulation, and the question arises as to what extent the results could be attributed to the specificity of the various agents used. Despite this concern, the transgene clearly altered the effects of exogenously administered angiotensin II, and therefore this work provides important information regarding interactions between angiotensin II and NO in the vasculature. This study is the first to directly demonstrate AT2 stimulation of vascular smooth muscle kininogenase activity, which, in turn, explains increased vascular production of bradykinin and NO in response to angiotensin II. Certainly, more work has do be done to verify and elucidate the physiologic importance of this angiotensin II/bradykinin/NO cascade and its relevance to heart failure, myocardial infarction, and vascular injury.

One intriguing notion that has evolved from this type of work is that failure of normal crosstalk between AT1 and AT2 may worsen development of cardiovascular disease. Thus, absence or decreased activity of AT2 may allow the deleterious effects of AT1 stimulation to go unchecked. Interestingly, NO has been reported to downregulate AT1 expression (17). Therefore, enhanced $\mathrm{NO}$ production in response to AT2 stimulation may diminish AT1 respon- siveness directly. Conversely, selective pharmacotherapeutic stimulation of the AT2 may have beneficial effects in treatment of cardiovascular diseases. Treatment with AT1 antagonists, which leave AT2 unblocked, may in part achieve this end. As the role of the AT2 rises from physiologic obscurity, a more thorough understanding of its interactions with the AT1 and the NO system is emerging. Understanding these interactions may allow greater insight into the full impact of angiotensin II in disease.

1. Ichiki, T., et al. 1995. Effects on blood pressure and exploratory behaviour of mice lacking angiotensin II type-2 receptor. Nature. 377:748-750

2. Hein, L., Barsh, G.S., Pratt, R.E., Dzau, V.J., and Kobilka, B.K. 1995. Behavioural and cardiovascular effects of disrupting the angiotensin II type2 receptor in mice [erratum 1996, 380:366]. Nature. 377:744-747.

3. Hutchinson, H.G., Hein, L., Fujinaga, M., and Pratt, R.E. 1999. Modulation of vascular development and injury by angiotensin II. Cardiovasc. Res. 41:689-700.

4. Nakajima, M., et al. 1995. The angiotensin II type 2 (AT2) receptor antagonizes the growth effects of the AT1 receptor: gain-of-function study using gene transfer. Proc. Natl. Acad. Sci. USA. 92:10663-10667.

5. Liu, Y.H., et al. 1997. Effects of angiotensin-converting enzyme inhibitors and angiotensin II type 1 receptor antagonists in rats with heart failure. Role of kinins and angiotensin II type 2 receptors. J. Clin. Invest. 99:1926-1935.

6. Ohkubo, N., et al. 1997. Angiotensin type 2 receptors are reexpressed by cardiac fibroblasts from failing myopathic hamster hearts and inhibit cell growth and fibrillar collagen metabolism. Circu- lation 96:3954-3962.

7. Nio, Y., Matsubara, H., Murasawa, S., Kanasaki, M., and Inada, M. 1995. Regulation of gene transcription of angiotensin II receptor subtypes in myocardial infarction. J. Clin. Invest. 95:46-54.

8. de Gasparo, M., and Siragy, H.M. 1999. The AT2 receptor: fact, fancy and fantasy. Regul. Pept. 81:11-24.

9. Horiuchi, M., Hayashida, W., Kambe, T., Yamada, T., and Dzau, V.J. 1997. Angiotensin type 2 receptor dephosphorylates $\mathrm{Bcl}-2$ by activating mitogen-activated protein kinase phosphatase- 1 and induces apoptosis. J. Biol. Chem. 272:19022-19026.

10. Yamada, T., Horiuchi, M., and Dzau, V.J. 1996. Angiotensin II type 2 receptor mediates programmed cell death. Proc. Natl. Acad. Sci. USA 93:156-160.

11. Munzenmaier, D.H., and Greene, A.S. 1996 Opposing actions of angiotensin II on microvascular growth and arterial blood pressure. Hypertension. 27:760-765.

12. Scheuer, D.A., and Perrone, M.H. 1993 Angiotensin type 2 receptors mediate depressor phase of biphasic pressure response to angiotensin. Am. J. Physiol. 264:R917-R923.

13. Siragy, H.M., and Carey, R.M. 1996. The subtype2 (AT2) angiotensin receptor regulates renal cyclic guanosine $3^{\prime}, 5^{\prime}$-monophosphate and AT1 receptor-mediated prostaglandin E2 production in conscious rats [see comments]. J. Clin. Invest. 97:1978-1982.

14. Siragy, H.M., and Carey, R.M. 1997. The subtype 2 (AT2) angiotensin receptor mediates renal production of nitric oxide in conscious rats. J. Clin. Invest. 100:264-269.

15. Gohlke, P., Pees, C., and Unger, T. 1998. AT2 receptor stimulation increases aortic cyclic GMP in SHRSP by a kinin-dependent mechanism. Hypertension. 31:349-355.

16. Tsutsumi, Y., et al. (1999). Angiotensin II type 2 receptor overexpression activates the vascular kinin system and causes vasodilation. J. Clin. Invest. 104:925-935

17. Ichiki, T., et al. 1998. Downregulation of angiotensin II type 1 receptor gene transcription by nitric oxide. Hypertension. 31:342-348. 